\title{
Tumors of the anterior visual pathway in pediatric patients
}

\author{
Chidambaram Balasubramaniam, Venkataraman Balasubramaniam
}

Kanchi Kamakoti CHILDS Trust Hospital Chennai, India

\begin{abstract}
This report is based on the experience in the management of 17 children with neoplasms involving the anterior visual pathway (i.e. the optic nerves and chiasm). Although gliomas are the commonest tumors other neoplasms are also seen in this location. Hence there is a need for histologic verification before all neoplasms seen here are managed as gliomas. Surgical excision without compromising the neurologic, visual and endocrine state is suggested as the main option. Chemotherapy and or radiation can be considered as adjuvants depending on the age of the patient. Radiation is best avoided in children less than four years of age. (J Pediatr Neurol 2003; 1(2): 89-94).
\end{abstract}

Key words: tumor, anterior visual pathway, chiasm gliomas.

\section{Introduction}

Tumors involving the anterior visual pathway (AVP) viz optic nerves and chiasm are not commonly seen in general neurosurgical work. Their incidence, however, increases if one considers only pediatric patients (age less than 20 years). There is a general tendency to group these tumors topographically and also from the pathological point of view at the same time. So much so in any discussion on tumors of

Correspondence: Chidambaram Balasubramaniam New No. 12 (Old. 26) Second Main Road, C.I.T.Colony, Mylapore,

Chennai-4.

India.

Tel: 0091-044-4992970.

E-mail: chidu@vsnl.com

Received: March 11, 2003.

Revised: May 21, 2003.

Accepted: May 26, 2003.
AVP only gliomata are discussed. Even now there is no consensus regarding the overall management of these patients. We are herewith reporting our experience with 17 cases.

\section{Materials and Methods}

During the years 1991-2001 17 histologically verified tumors involving the AVP were seen and operated upon in pediatric patients. Of these two cases of medulloepithelioma have been detailed in separate papers earlier $(1,2)$. Of the 17 , seven were males and ten females. The age distribution is given in Table 1. The youngest was four months old and the oldest fifteen years.

The duration of symptoms varied, with the maximum being five years. The shortest was 12 days. The symptoms were mainly proptosis and loss of vision. They are detailed in Table 2. In one patient there was no visual loss. In one patient aged four months it was not possible to assess the degree of visual impairment. Two patients had neurofibromatosis type 1 (NF-1). Visual evaluation was done only by confrontation. We have no facilities to do visual evoked patential. The location of the lesions is given in Table 3. Computerized tomography (CT) and magnetic resonance imaging (MRI) were done on all these patients. After appropriate ancillary tests they were operated upon. Histological confirmation was obtained in all patients (Table 4). The detailed description of all the gliomas is given in Table 5 .

The operative approach was based on the location and spread of the lesion. Using a bicoronal skin flap and a unilateral free bone flap, subfrontal exploration was carried out in 6 cases. Frontotemporal craniotomy was done in 2 cases. Orbitofrontal craniotomy was done in 6 cases. A pterional approach was used in 3 cases. The extent of resection is given Table 6. Ipsilateral tarsorraphy was done as a routine in all cases. Two cases were treated with chemotherapy; one of these had radiation also. In ten cases post operative radiation was employed. 
Table 1. Showing age distribution

\begin{tabular}{ll}
\hline $\begin{array}{l}\text { Ages } \\
\text { (years) }\end{array}$ & n \\
\hline $0-5$ & 10 \\
$>5-10$ & 5 \\
$>10-15$ & 2 \\
$>15-20$ & 0 \\
\hline \hline
\end{tabular}

Table 2. Showing the various symptoms

\begin{tabular}{ll}
\hline \hline Symptoms & $\mathbf{n}$ \\
\hline Unilateral visual impairment or loss & 10 \\
Bilateral visual impairment or loss & 6 \\
Proptosis & 7 \\
Endocrine symptoms & 3 \\
Headache & \\
\hline \hline
\end{tabular}

Table 3. Showing location of lesions

\begin{tabular}{ll}
\hline \hline Localizations & n \\
\hline Suprasellar and chiasm & 9 \\
One optic nerve with or without & \\
involvement of intracranial part & 7 \\
Optic nerve and chiasm & 1 \\
\hline \hline
\end{tabular}

Table 4. Showing pathology of the lesions

\begin{tabular}{ll}
\hline \hline Pathologies & n \\
\hline Glioma & 14 \\
Medulloepithelioma & 2 \\
Ganglionglioma & 1 \\
\hline \hline
\end{tabular}

\section{Results}

Two patients died within a year. Of these one patient (Case 4) died within a week following surgery. The second patient (Case 7) died within a year. Both of them had medulloepithelioma. Two were lost to follow up after six months. The others are doing well. Of these, one patient who was advised radiation but did not undergo the radiotherapy. He returned after 2 years because of continued growth and had to be operated again. Postoperatively ten patients had diabetes insipidus for a variable duration. This was treated. Only one patient continues to have diabetes insipidus and is on therapy. One patient who had a chiasmal and optic nerve glioma had hydrocephalus when he came in. He first had a ventriculo peritoneal shunt before surgery for the tumor. Then he was operated for the tumor. Later because of development of ascites the shunt was converted into a ventriculo atrial shunt. This case has also been reported (3) the results correlated with extent of resection and pathology are given Table 6 .
Table 6. Showing extent of resection

\begin{tabular}{ll}
\hline Resections & n \\
\hline Total resection & 5 \\
Near total resection $(>90 \%)$ & 1 \\
Subtotal resection $(70-90 \%)$ & 2 \\
Partial resection $(11-50 \%)$ & 9 \\
& \\
\hline \hline
\end{tabular}

\section{Effect on vision}

In seven patients where the lesion, though extensive, was confined to the optic nerve the nerve had to be sacrificed. In patients in whom the lesion was confined to one optic nerve the vision though lost in that eye continued to be good in the other eye. In one patient who had no vision in one eye and good vision in the other eye, there was slight temporary postoperative diminution in visual acuity in the healthy eye. But this improved. She had a suprasellar chiasmatic glioma which was involving one optic nerve also. There was no worsening of the vision in any other patient.

\section{Effect on proptosis}

Of the seven who had proptosis there was considerable improvement and appreciable regression of the proptosis.

\section{Discussion}

Tumors involving the AVP are not very common; so much so individual experience is limited to a few cases. Sulton and Molloy quote from other reports as follows "...the incidence ranges from $0.84 \%$ of brain tumors in all age groups to $3-6 \%$ of tumors in paediatric age group". They also mention that of these $20 \%$ had (NF-1) (4). In our series of one hundred consecutive histologically verified tumors of the central nervous system in patients less than 20 years of age, seventeen cases of tumors of AVP have been encountered. In this report the cases were collected based on their location (and not on histological grounds). Generally the tumors of this region are lumped together. In any discussion only the point of view of pathology viz gliomas is considered. But many other tumors also arise in this region. Our main reason for this report is that we felt that many different types of neoplasms (and even non neoplastic conditions) occur in this area. And it is not very correct to assume that all lesions are gliomata without histological confirmation.However, it cannot be denied the gliomata are by far commonest. In our series out of 17 cases fourteen patients had gliomata (82\%).

The signs and symptoms depend on the location of the lesion and not the pathology of the lesion. In this series visual loss and/or proptosis had been the problems that brought the patient to the doctor. But 
Table 5. The description of all the gliomas

Case 1: Pilocytic astrocytoma of the optic nerve. The resected margin is free of tumor. The tumor shows significant neovascularisation. There is no plemorphism or mitosis.

Case 2: Shows a tumor composed of proliferating neoplastic astrocytes. These astrocytes have thick fibres and hence the tumor has a dense fibrillary back ground. Many Rosenthal fibres and colloid bodies are seen. Phosphotungstic acid and haemotoxylin stain (for glia) confirms the astrocytic nature of the tumor.

Case 3: Shows the tumor has a loose, lacy appearance due to microcystic change. The tumor is composed of fibrillary astrocytes. Many of these have thick and prominent processes. Occasional Rosenthal fibres are seen. Perivascular arrangement of tumor cells are seen. There is no pleomorphism or mitotic activity. The adjacent brain parenchyma shows infiltration in the peripheral part.

Case 6: Pilocytic astrocytoma arising from intra orbital portion of the optic nerve. The Intracranial portion of the optic nerve showed only gliosis, but could represent extension of the lesion located anteriorly.

Case 8: Pilocytic astrocytoma (optic chiasm).

Case 9: The material submitted shows a glioma, with active fibre formation and Rosenthal fibres. The glioma cells show moderate degree of pleomorphism, but mitosis, angiogenesis and necrosis are not evident.

Case 10: The lesion shows a tumor composed of both astrocytic and oligodendroglial components. The tumor cells show minimal pleomorphism. At places, there are areas of calcification and vascular proliferation. In other areas, the tumor has a whorling pattern that resembles the nerve fasicles. The enclosing dural sheath appears normal. Section from the intracranial part of the tumor shows predominantly a pilocytic type of astrocytoma with many hyalinised vessels and few Rosenthal fibres. There is no pleomorphism or evidence of mitotic activity.

Case 11: Shows a tumor composed of proliferating fibrillary astrocytes. The tumor cells are spindle shaped and have prominent glial processes. Microcystic degeneration is noted within the tumor. Occasional Rosenthal fibres are seen. The vascular channels are prominent. Occasional large astrocytes with autophagic vacuoles are seen.

Case 12: The resected optic nerve shows thickening of the pial septae, hyalinisation of vessel walls and total gliosis of a few of the optic nerve fasicles. The resected lesion is pilocytic astrocytoma, with neovascularisation and formation of glomerular structures. The tumor has invaded the nerve totally effacing the structure.

Case 13: The material shows spindle shaped cellular tumor. Glial fibres are arising from them. At places mass of colloid bodies, Rosenthal fibres are seen, indicating the glial origin and chromicity. The tumor cells reveal variable pleomorphism and focal increased mitotic activity. The histological features are suggestive of astrocytoma Gr II. No areas of necrosis or neovascularisation are noted.

Case 14: Optic nerve glioma, infiltrating the nerve pilocytic type.

Case 15: The tumor resected shows a biphasic picture of fasicular arrangement of spindle shaped pilocytic astrocytes and islands of loosely arranged astrocytoma cells with microcystic change. The tumor is moderately vascular. In one portion the tumor is traversed by thin fibrous septae into nodular compartments, suggesting the origin of the tumor from opto-chiasmatic zone. There is no pleomorphism. Numerous Rosenthal fibres are seen.

Case 16: Section from the optic nerve shows enlargement and replacement of funicles by a glial neoplasm composed of fibrillary astrocytes. The tumor cells are spindle shaped, show minimal pleomorphism and stroma is densely fibrillated. Adjacent to highly fibrillated areas are zones with prominent microcystic change. Few Rosenthal fibres are evident. The tumor is well walled off by the arachnoid and dural collagen. The cranial end of the nerve also shows similar morphology.

Case 18: Section shows transverse and longitidunal sections of the optic nerve. The chiasmal end is markedly enlarged. There is diffuse enlargement of the funicles with partial disruption due to infiltration by a highly fibrillated, paucicellular astrocytic neoplasm. The nuclei are small, oval to elongated, vesicular and bland. Numerous eosinophilic Rosental fibers are seen. Perivascular hyalinization is also noted. In addition to the funicular infiltration, the tumor is seen encircling the nerve in the subpial and subarachnoid plane. There are no mitoses or necrosis. 
Table 7. Showing correlation of extent of resection and pathology with report

\begin{tabular}{llll}
\hline \hline Case no & Extent of resection & Pathology & Prognosis \\
\hline 1 & $\mathrm{~T}$ & Glioma & All right \\
2 & $\mathrm{NT}$ & Glioma & All right \\
3 & $\mathrm{P}$ & Glioma & All right \\
4 & $\mathrm{P}$ & Medulloepithelioma & Died in post operative period \\
5 & $\mathrm{P}$ & Ganglionglioma & All right \\
6 & $\mathrm{~T}$ & Glioma & All right \\
7 & $\mathrm{P}$ & Medulloepithelioma & Died within a year \\
8 & $\mathrm{P}$ & Glioma & Lost for follow up \\
9 & $\mathrm{P}$ & Glioma & All right \\
10 & $\mathrm{~T}$ & Glioma & All right \\
11 & $\mathrm{P}$ & Glioma & All right \\
12 & $\mathrm{ST}$ & Glioma & All right \\
13 & $\mathrm{P}$ & Glioma & Lost for follow up \\
14 & $\mathrm{P}$ & Glioma & All right \\
15 & $\mathrm{ST}$ & Glioma & All right \\
16 & $\mathrm{P}$ & Glioma & All right \\
17 & $\mathrm{P}$ & Glioma & All right \\
\hline \hline
\end{tabular}

T: Total; NT: Near total; ST: Subtotal; P: Partial.

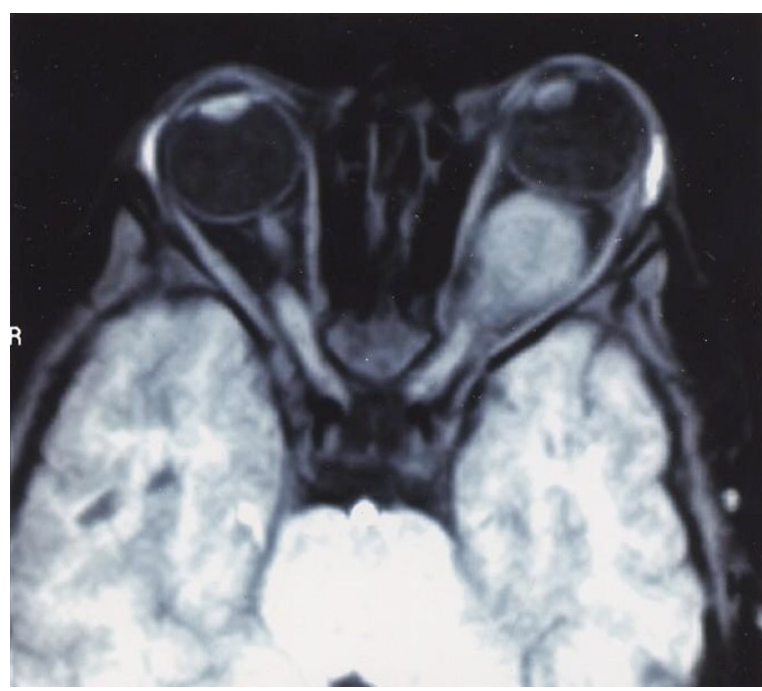

Figure 1. MR scan showing optic nerve glioma.

the visual loss is extensive by the time the patient comes to the surgeon. This is because even a total depends on the pathological findings. Some would loss of vision in one eye goes undetected on account of the young age of the patient.

Proptosis occurs late in time and this is often accompanied by almost total loss of vision in that eye. This prevailed in 7 cases. In one case the loss of vision was total in one eye and in the other eye there was a temporal field loss. This was a suprasellar lesion. Some times the visual loss is discovered when the patient is brought for proptosis. The overall management of these lesions assume the lesion to be a glioma whether there is NF-1 or not; and they manage it according to their philosophy. For example Takeuchi et al (5) state that "if the patient has concurrent NF-1 or if the optic nerve and its radiations are involved the mass can be assumed to be an astrocytoma and surgical intervention is not (emphasis authors') required to establish the diagnosis". In our series only two patients had NF-1 and one of these had medulloepithelioma.

As against this can be quoted the experience of Cohen et al (6) where a diagnosis of glioma was made in a patient where the chiasm was involved and radiation given in an appropriate manner. Later at postmortem this turned out to be a germinoma. In a frank and honest appraisal the authors state "Death might have been prevented had the correct histological diagnosis been made by biopsy and entire ventricular system irradiated" (6).

There are no diagnostic clinical or radiological criteria that would help to arrive at a correct conclusion regarding the pathology. Even in patients with NF-I the diagnosis of glioma can at best be by presumption. In two of our cases the clinical and radiological findings were similar to other cases of glioma yet they turned out to be medullo epithelioma. And one other case with similar radiological pictures turned to be a ganglionglioma (Figures 1, 2a, and 2b).

So we feel that all cases must have the pathology established by surgery. It must be remembered that even at operation at times one cannot be sure about the nature of the tumour. This brings us to the question, that even if one assumes that the lesion is glioma what should be the line of treatment. Here again there is no consensus. Some feel that if the lesion is confined to one optic nerve and if the proptosis is minimal (or nil) nothing need be done. Their argument is based on the hypothesis that progression of the tumor to the intracranial part and chaisma is almost nil. In the series of Alvord and Lofton (7) out of 99 optic nerve glioma without 


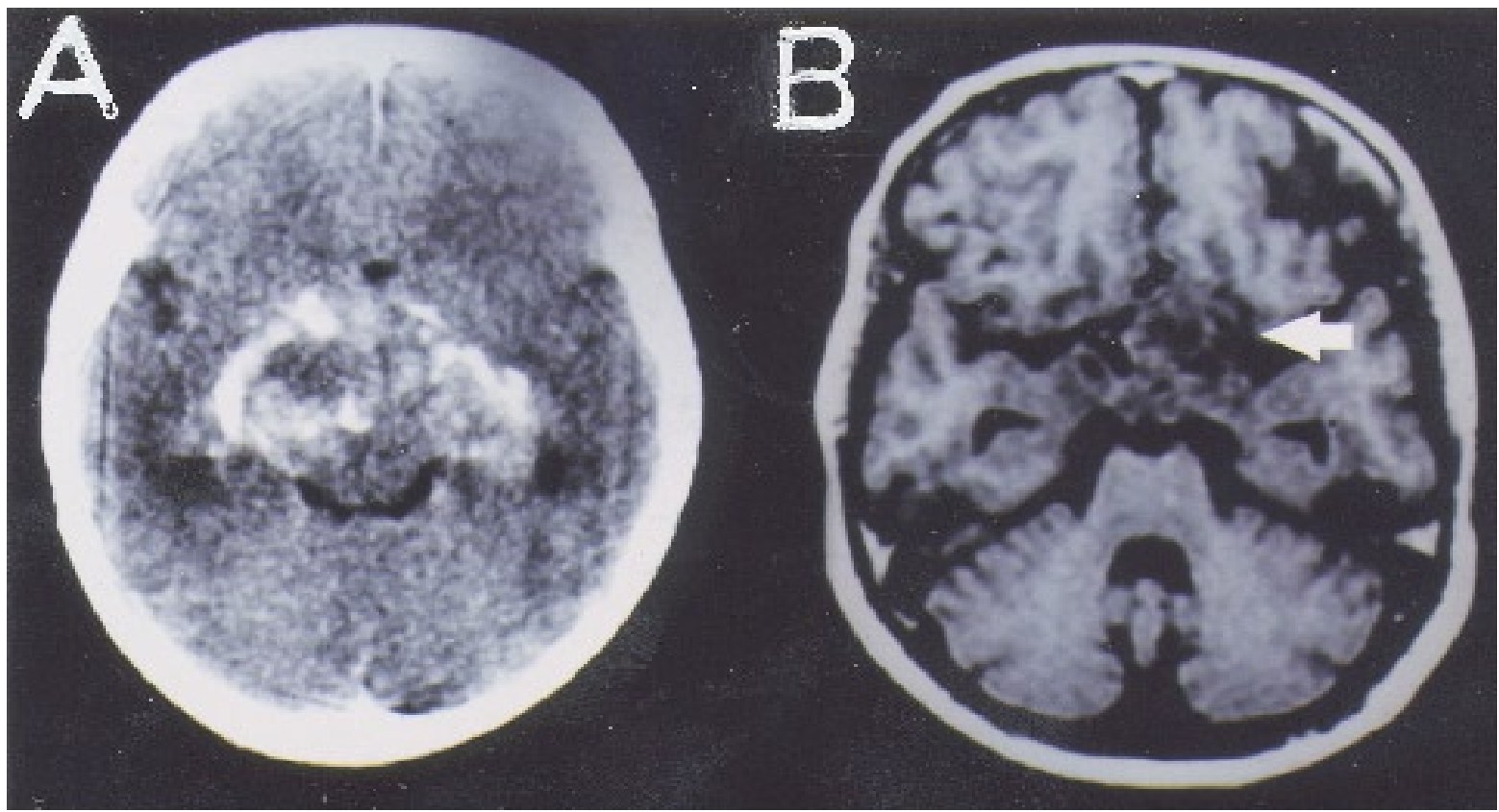

Figures $\mathbf{2 a}$ and $\mathbf{2 b}$. CT and MR of the child with ganglion glioma (arrow in the MRI points to the chiasmal location of the tumor).

any intracranial extension only in 13 the growth recurred in the nerve. In six the tumor had spread to the chiasm (7). These authors state that, "their (gliomas of optic nerve or chiasm) natural history has been debated for almost a century, with some investigators believing that they are not neoplasms but hamartomas (that is they are congenital malformations and do not grow continuously). Their treatment had been similarly debated and some authors consider that little or no treatment is successful as radiation therapy or radical surgical excision". The authors conclude that surgical excision is better than irradiation in terms of recurrence of optic nerve glioma but not obviously in terms of retention of vision (7).

If we assume that the lesion is a glioma, the consensus today seems to be in favor of as extensive a resection as is possible and as is safe. As we approach the midline the chances for extensive resection recede and to that extent the prognosis is dim.

This limiting factor is common to all kinds of tumors. Johannson et al (8) in analysis 14 cases of ganglionglioma state that "the tumors arising in the midline carry a poor prognosis and result in a significant neurological deficit, and no therapeutic recommendation is possible in this group".

On the other hand while reporting on an experience of 54 cases of optic pathway glioma Lancon et al (9) write "tumor enhancement and presentation prior to 3 years of age were associated with tumor progression" and "hypothalamic involvement did not influence progression free survival except in children less than 3 years of age".
Generally the opinion is that these tumors are best treated by surgery. However, there are some who feel surgery may be deferred or even avoided in those with NF-1.

Our own policy is to perform the surgery on the premise the lesion is a glioma. For the tumors as radical a resection as is possible and safe is done. Where one has removed a lesion totally as shown by postoperative CT (done within 24-48 hrs) no further treatment is needed. But where the resection of a glioma is incomplete (because of various factors and in children less than 3-4 years) chemotherapy is used as a supplementary measure. When the patient is older than 4 years then chemotherapy and radiation may be used individually or in combination. In all cases prognosis has to be considered from two angles a) preservation of vision and b) prolongation of life. For recurrences we prefer surgery.

Preservation of vision in the affected eye is at present impossible. One can however prevent a spread of disease through the chiasm to the unaffected eye. Regarding prolongation of life the outcome depends on the histological findings.

\section{Conclusion}

We feel that a histological diagnosis is mandatory in every case irrespective of the amount of circumstantial evidence pointing to the diagnosis of glioma. Operative resections must be based on a provisional diagnosis of glioma. The post surgery treatment can be done on the basis of histology and age of the patient. If one feels that extensive resection is unjustified then histological proof must however be obtained. This can be done by open surgery or by stereotactic techniques. 


\section{References}

1. Biswas J, Bushan B, Jayakumar N, Chidambaram B. Teratoid malignant medulloepithelioma of the optic nerve: report of a case and review the literature. Orbit 1999; 18: 191-196.

2. Chidambaram B, Santhosh V. Balasubramaniam V. Medulloepithelioma of the optic nerve with intradural extension - report of two cases and a review of the literature. Child's Nerv Syst 2000; 16: 329-333.

3. Chidambaram B, Balasubramaniam V. CSF ascites: a rare complication of ventriculoperitoneal shunt surgery. Neurol India 2000; 48: 378-380.

4. Sulton LN, Molloy P. Pediatric Neurosurgery. Surgery of the Developing Nervous System (4th ed). Philadephia: WB Saunders, 2001.

5. Takenchi H, Kabuto M, Sato K, Kubota T. Chiasmal gliomas with spontaneous regression: proliferation and apoptosis. Child's Nerv Syst 1997; 13: 229-233.

6. Cohen DN, Steinberg M, Buchwald R. Suprasellar germinomas: diagnostic confusion with optic glioma. Case report. J Neurosurg 1974; 41: 490493.

7. Alvord EC Jr, Lofton S. Glioma of the optic nerve or chiasm: outcome by patients' age, tumor site, and treatment. J Neurosurg 1988; 68: 85-98.

8. Johannsson JH, Rekate HL, Roessonann U. Ganglion glioma pathology and clinical correlation. J Neurosurg 1981; 54: 58-63.

9. Lancon J, Doxey D, Shapiro K, Swift D, Sklar F, Bruce DA. Optic pathway gliomas. a review of 53 cases. Child's Nerv Syst 1998; 15: 422 (abstract). 